
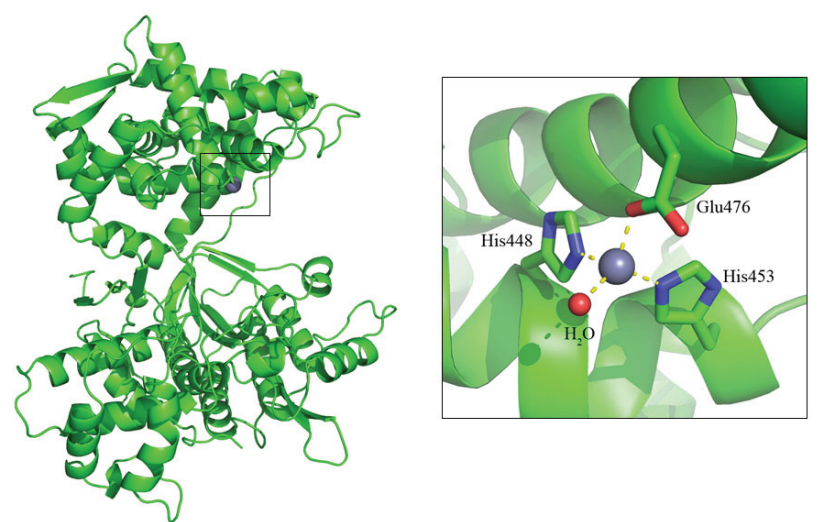

Figure 1. Overview of the BtDPP III structure. A two domains protein with characteristic M49 fold. The active site is located in the upper domain, containing a zinc ion coordinated by two histidines from the characteristic HECLGH motive, one glutamic acid from the EEARAD motive, and a water molecule.

Keywords: Dipeptidyl peptidase III, metallopeptidase, M49 family, Bacteroides thetaiotaomicron, Caldithrix abyssi

\section{MS5-P9 The cell-shape determining Csd6 protein from Helicobacter pylori constitutes a new family of L,D-carboxypeptidase \\ Hyoun Sook Kim ${ }^{1,2}$, Byung Woo Han ${ }^{1}$, Se Won Suh ${ }^{2}$}

1. Research Institute of Pharmaceutical Sciences, College of Pharmacy, Seoul National University, Seoul 151 742, Republic of Korea

2. Department of Chemistry, College of Natural Sciences, Seoul National University, Seoul 151 742, Republic of Korea

email: kobooks@gmail.com

Helicobacter pylori causes gastrointestinal diseases, including gastric cancer. Its high motility in the viscous gastric mucosa facilitates colonization of the human stomach, and depends on the helical cell shape and the flagella. In $H$. pylori, Csd6 is one of the cell shape-determining proteins that play key roles in determining the cell shape by alteration of cross-linking or by trimming of peptidoglycan muropeptides. Csd6 is also involved in deglycosylation of the flagellar protein FlaA. To better understand its function, we have carried out biochemical, biophysical, and structural characterizations. We show that Csd6 has three-domain architecture and exists as dimers in solution. The $\mathrm{N}$-terminal domain plays a key role in dimerization. The middle catalytic domain resembles those of L,D-transpeptidases and its pocket-shaped active-site is uniquely defined by the four loops I-IV, among which the loops I and III show the most distinct variations from the known L,D-transpeptidases. Our mass analyses confirm that Csd6 functions only as L,D-carboxypeptidase but not as L,D-transpeptidase. Our D-Ala-complexed structure reveals binding modes of both the substrate and product to the catalytic domain. The C-terminal nuclear transport factor 2-like domain possesses a deep pocket for possible binding of pseudaminic acid and our in silico docking supports its role in deglycosylation of flagellin. On the basis of our findings, we propose that $H$. pylori Csd6 and its homologs constitute a new family of L,D-carboxypeptidase. This work provides insights into the function of Csd6 in regulating the helical cell shape and motility of $H$. pylori.

Keywords: Csd6, cell shape, L,D-carboxypeptidase, Helicobacter pylori, HP0518, flagellin, peptidoglycan 